\title{
Genomic Profiles of De Novo High- and Low-Volume Metastatic Prostate Cancer: Results From a 2-Stage Feasibility and Prevalence Study in the STAMPEDE Trial
}

Clare Gilson, MRCP, MD ; Fiona Ingleby, PhD ${ }^{1}$; Duncan C. Gilbert, PhD ${ }^{1}$; Marina A. Parry, PhD²; Nafisah B. Atako, MSc ${ }^{1}$; Adnan Ali, MBBS ${ }^{3}$; Alex Hoyle, MRCS, MD ${ }^{3,4}$; Noel W. Clarke, MBBS, ChM ${ }^{3,4}$; Melissa Gannon, MSc ${ }^{1}$; Chris Wanstall, BSc ${ }^{1}$; Christopher Brawley, MSc ${ }^{1}$; Malcolm D. Mason, MD ${ }^{5}$; Zafar Malik, FRCR ${ }^{6}$; Andrew Simmons, PhD ; Andrea Loehr, PhD; Alison Parry-Jones, PhD ${ }^{5}$; Rosalind Eeles, MBBS, PhD ${ }^{8,9}$; Zsofia Kote-Jarai, PhD ${ }^{9}$; Nicholas D. James, MBBS, PhD ${ }^{9}$ Claire Amos, PhD Mahesh K. B. Parmar, PhD ${ }^{1}$; Ruth E. Langley, MD, PhD ${ }^{1}$; Matthew R. Sydes, MSc ${ }^{1}$; Gerhardt Attard, MD, PhD ${ }^{2}$; and Simon Chowdhury, MD, $\mathrm{PhD}^{10}$; for the STAMPEDE Investigators

PURPOSE The STAMPEDE trial recruits men with newly diagnosed, high-risk, hormone-sensitive prostate cancer. To ascertain the feasibility of targeted next-generation sequencing (tNGS) and the prevalence of baseline genomic aberrations, we sequenced tumor and germline DNA from patients with metastatic prostate cancer (mPCa) starting long-term androgen-deprivation therapy (ADT).

METHODS In a 2-stage approach, archival, formalin-fixed, paraffin-embedded (FFPE) prostate tumor core biopsy samples were retrospectively subjected to 2 tNGS assays. Prospective enrollment enabled validation using tNGS in tumor and germline DNA.

RESULTS In stage 1, tNGS data were obtained from 185 tumors from 287 patients (65\%); $98 \%$ had de novo mPCa. We observed PI3K pathway aberrations in 43\%, due to PTEN copy-number loss (34\%) and/or activating mutations in PIK3 genes or AKT (18\%) and TP53 mutation or loss in 33\%. No androgen receptor (AR) aberrations were detected; $R B 1$ loss was observed in $<1 \%$. In stage 2, $93(92 \%)$ of 101 FFPE tumors (biopsy obtained within 8 months) were successfully sequenced prospectively. The prevalence of DNA damage repair (DDR) deficiency was $14 \%$ (somatic) and $5 \%$ (germline). BRCA2 mutations and mismatch repair gene mutations were exclusive to high-volume disease. Aberrant DDR (22\% v 15\%), Wnt pathway (16\% v4\%), and chromatin remodeling ( $16 \% \mathrm{v} 8 \%$ ) were all more common in high-volume compared with low-volume disease, but the small numbers limited statistical comparisons.

CONCLUSION Prospective genomic characterization is feasible using residual diagnostic tumor samples and reveals that the genomic landscapes of de novo high-volume mPCa and advanced metastatic prostate cancer have notable similarities (PI3K pathway, DDR, Wnt, chromatin remodeling) and differences ( $A R, R B 1$ ). These results will inform the design and conduct of biomarker-directed trials in men with metastatic hormone-sensitive prostate cancer.

JCO Precis Oncol 4:882-897. @ 2020 by American Society of Clinical Oncology

ASSOCIATED

CONTENT

Appendix

Author affiliations

and support

information (if

applicable) appear at the end of this

article.

Accepted on May 22, 2020 and published at ascopubs.org/journal/ po on July 28,2020 : DOI https://doi.org/10. 1200/P0.19.00388

\section{INTRODUCTION}

Although localized prostate cancer is often an indolent disease, metastatic prostate cancer is usually lethal. ${ }^{1}$ Significant improvements in outcome for men with metastatic disease are achieved when effective systemic therapies are used early, shortly after commencing androgen-deprivation therapy (ADT), termed hormone-sensitive disease. Docetaxel, abiraterone, enzalutamide, and apalutamide all have level 1 evidence showing improved overall survival when used in addition to ADT in metastatic hormone-sensitive prostate cancer (mHSPC). ${ }^{2-6}$ However, we lack predictive biomarkers.
Next-generation sequencing (NGS) studies of metastatic castrate-resistant prostate cancer (mCRPC), defined as progressive disease despite ADT, have identified important therapeutically targetable aberrant pathways. ${ }^{7-12}$ These studies used biopsies (mostly lymph node, liver, or bone metastases) and notably identified genomic aberrations in DNA damage repair (DDR) pathways leading to the development of PARP inhibitors for DDR-deficient mCRPC. ${ }^{13}$ To date, the majority of NGS analyses linked to clinical data of untreated cancers have focused on low- to intermediate-risk cohorts (eg, TCGA), ${ }^{9,14,15}$ whereas the profile of $\mathrm{mCRPC}$ has been 


\section{CONTEXT}

\section{Key Objective}

In order to characterize the genomic profile of de novo metastatic prostate cancer, we conducted a feasibility and prevalence study using diagnostic formalin-fixed paraffin-embedded (FFPE) prostate core biopsies from participants enrolled in STAMPEDE clinical trial, a proportion of whom also provided germline DNA.

\section{Knowledge Generated}

We demonstrate that it is feasible to perform tNGS using archival samples and the highest success rates are observed when sequencing is performed within 8 months of biopsy fixation. We show the profile of de novo metastatic prostate cancer is distinct from that seen in metastatic castrate resistant disease (mCRPC) and prostatectomy cohorts. Unique to our study, we show baseline genomic differences according to metastatic burden with high volume metastatic disease showing most similarities to metastatic castrate resistant prostate (mCRPC), with the exception of AR mutations which are absent and Rb1 loss which is rare.

\section{Relevance}

These data will inform the design of biomarker-directed trials in de novo metastatic hormone sensitive prostate cancer.

informed by autopsy studies, ${ }^{11,16}$ pooled clinical trial cohorts (eg, SU2C), ${ }^{12}$ and single-center clinical cohorts (eg, MSK-IMPACT). ${ }^{7}$ When included, patients with $\mathrm{mHSPC}$ have often had relapsed disease after treatment of localized prostate cancer, and there are relatively limited data specific to de novo mHSPC. ${ }^{7}$

STAMPEDE is an adaptive, multi-arm, multistage platform protocol that seeks to evaluate therapeutic strategies in newly diagnosed high-risk or metastatic prostate cancer. Our aim in this study was to support the implementation of biomarker-stratified treatment in $\mathrm{mHSPC}$. We hypothesized that the genomic profile of mHSPC would differ from localized and advanced prostate cancer, necessitating specific prevalence data to inform trial design. We performed a 2-stage study in which we piloted targeted nextgeneration sequencing (tNGS) using routinely available prostate cancer samples in a subset of STAMPEDE trial participants, some of whom also underwent germline testing and volumetric assessment of metastatic disease burden. We sought to evaluate the feasibility of tNGS, assess the prevalence of baseline genomic aberrations in mHSPC, and, where it was known, explore differences according to metastatic burden.

\section{METHODS}

Stage 1 was a retrospective analysis using a dedicated protocol reviewed by the West Midlands Regional Ethics Committee, United Kingdom (16/WM/0188) applied to patients who had already consented to gift their diagnostic samples when trial consent was obtained. Stage 2 was a prospective study undertaken following a STAMPEDE protocol amendment (v16) to evaluate the feasibility of obtaining tumor and germline DNA before trial randomization and to validate the results from stage 1 . Study enrollment was an optional aspect of trial participation and required specific additional consent. The STAMPEDE eligibility criteria are described in full elsewhere. ${ }^{1,3,6,17}$ Briefly, all participants included in this study were starting longterm ADT for metastatic disease, had confirmed prostate adenocarcinoma, and were fit to receive the experimental treatments assessed in STAMPEDE. Residual diagnostic samples were used, which in stage 2 must have been obtained within 8 months of study registration; no extra biopsies were required. With the permission of the trial oversight groups, baseline clinical characteristics are presented for all randomly assigned participants.

\section{Stage 1}

Residual diagnostic pretreatment archival formalin-fixed paraffin-embedded (FFPE) biopsy samples were retrospectively retrieved from consenting participants randomly assigned between November 2011 and May 2017. Pathologic review of a hematoxylin and eosin-stained slides confirmed the histologic diagnosis and estimated tumor content. Where multiple FFPE blocks were available per patient, the sample with the highest tumor content was selected. In stage 1 , one sample per patient was sequenced in one of two laboratories (Fig 1). A total of 186 samples were sequenced in a US clinically accredited laboratory (Foundation Medicine) and are referred to as cohort 1a. The Foundation Medicine T7 hybrid-capture assay includes 395 cancer-related genes and reports singlenucleotide variants (SNVS), indels, copy-number alterations, and selected rearrangements. ${ }^{18}$ We here report the pathogenic changes that occurred in 39 genes shown to be aberrant in prostate cancer on the basis of prior publications. $^{7,12}$ To evaluate implementing screening within STAMPEDE UK centers, we submitted samples to a UK-based laboratory using the TST-170 panel (Illumina) delivered by Almac Diagnostics, (Craigavon, UK), referred to as cohort 1b. The TST170 uses a hybrid-capture method to extract DNA and RNA to detect SNVs, indels, selected rearrangements, and fusions. ${ }^{19,20}$ Herein we report pathogenic changes that occurred in 14 genes involved in DDR 


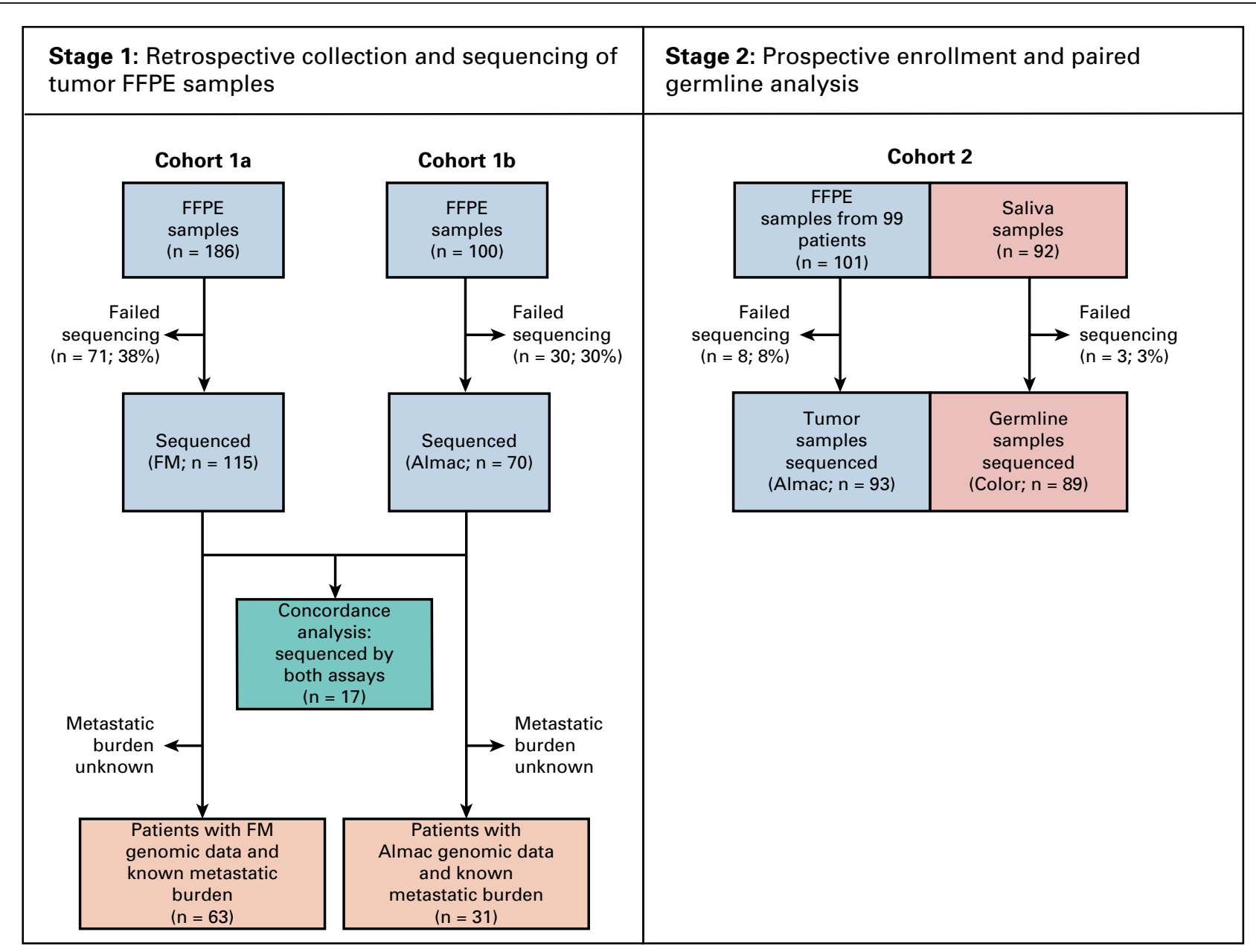

FIG 1. Overview of sampling approach. In cohort 1, the vast majority of samples were transrectal prostate biopsies; however, there were two metastatic biopsies submitted, including one bone biopsy. In cohort 2, all samples were transrectal prostate biopsies except for two lymph node biopsies. FFPE, formalin-fixed, paraffin-embedded; FM, Foundation Medicine.

selected based on evidence that they may predict sensitivity to PARP inhibition. ${ }^{13,21}$ Assay concordance was assessed for a subset of cases by comparing the tNGS results on the same DNA from both providers. Appendix Table A1 (online only) lists genes covered by each assay.

\section{Stage 2}

We undertook a prospective study to validate the retrospective analysis and assess the feasibility of implementing molecular characterization within the STAMPEDE trial, specifically focusing on identifying DDR pathway alterations to determine the feasibility of evaluating a PARP inhibitor. Additional substudy consent was obtained to retrieve residual diagnostic tumor samples and to provide a saliva sample for germline analysis. Both samples needed to be submitted within 10 weeks after commencing hormone therapy to adhere to the maximum prior hormone therapy permitted by the trial. All tumor samples (maximum of 2 per patient) must have been obtained within 8 months before registration. We subjected tumors to tNGS in a UK clinical laboratory (Almac Diagnostics, Craigavon, UK) and saliva to a clinically accredited 30-gene panel (Color Genomics). ${ }^{22}$ Ten genes involved in DDR were tested in both tumor and germline DNA; gene lists are provided in Appendix Table A1. Clinically relevant germline and somatic results were reported back to treating oncology teams to ensure participants were notified in accordance with participant information and consent.

Each study population was compared with the comparable intention-to-treat (ITT) population, defined as patients meeting the same eligibility criteria randomly assigned contemporaneously during this period at the same sites. Comparative analyses are limited to baseline characteristics, and the $\chi^{2}$ test was used with a false discovery rate correction applied within each characteristic. We have previously reported analyses by metastatic burden at presentation assessed using whole-body technetium bone scans and computed tomography or magnetic resonance 
imaging scans of the chest, abdomen, and pelvis, using the classification criteria used by the CHAARTED trial (ClinicalTrials.gov identifier: NCT00309985). ${ }^{5,23-25}$ An exploratory analysis of baseline genomic characteristics according to metastatic burden was performed for patients included in our analyses (Fig 1), and the Fisher's exact test was used to compare the frequency of specific mutations in high- and low-volume disease.

\section{RESULTS}

\section{Stage 1: Retrieval of Tissue From Men Randomly Assigned in the STAMPEDE Trial}

Cohort 1 included samples from 286 participants randomly identified from patients recruited between November 2011 and May 2017. A total of 186 samples were processed by Foundation Medicine (cohort 1a), of which 115 were successfully sequenced (62\%). One hundred samples were processed by Almac Diagnostics (cohort 1b), of which 70 were successfully sequenced (70\%); see Appendix Figure A1 for reasons for failure. Overall, samples were successfully sequenced for 185 (65\%) of 286 patients. Of these $185,98 \%$ had de novo metastatic disease and $62 \%$ had metastases to bone only, reflecting the typical disease distribution. As shown in Table 1 and Appendix Table A2 (online only), the sequenced population was representative of the comparable ITT trial cohort.

\section{Stage 2: Prospective Collection of Tissue and Saliva for Molecular Testing}

Cohort 2 contained 99 participants registered between December 2017 and August 2018 at 24 of the 107 STAMPEDE centers in the United Kingdom. Eleven of the 99 participants did not proceed to random assignment, but the baseline clinical characteristics (Table 1) are similar to cohort 1. Overall, they are representative of the comparable ITT trial population, although all had de novo metastatic disease, compared with $95 \%$ in the ITT. A total of 148 FFPE tumor blocks were obtained from 99 patients. In each case, the most suitable sample was selected after central pathology review (Almac Diagnostics, Craigavon, UK). If the first processed sample failed, a second sample was used where available. In total, 101 samples were processed, and overall sequencing success rate was 92\% (93 of 101), providing data for 93 of 99 patients. Saliva samples for germline DNA analysis were also collected for 92 of 99 patients, and 89 of 92 were successfully sequenced (Color Genomics).

\section{Genomic Landscape of mHSPC}

As shown in Figure 2, cohort 1a included 115 patients sequenced by Foundation Medicine, and the most prevalent aberrations were copy-number alterations or somatic point mutations in TP53 or PTEN. As would be expected for a population not previously treated with $A D T$, no androgen receptor $(A R)$ aberrations were detected. TP53 mutation or loss occurred in $33 \%$ of patients. Overall, PTEN copy-number alterations (25\%) or mutations (9\%) were observed in 34\%. PI3K pathway aberrations were detected in $18 \%$, occurring because of an activating mutation (13\%) or copy-number alteration (4\%) or PIK3R1 rearrangements (1.3\%). PI3K/AKT pathway aberrations occurred with PTEN loss in 9\% and without in 9\%. Wnt pathway aberrations occurred in $14 \%$, including mutation $(6 \%)$ or rearrangement (1\%) in APC (7\%) or mutations in CTNNB1 (7\%). Figure 3 shows the prevalence of alterations in 34 out of the 39 genes of interest on the basis of previous publications at both a gene and pathway level., ${ }^{72,26}$ Overall, aberrations were detected in $85 \%$ of patients, with $56 \%$ harboring $\geq 2$ and $24 \% \geq 3$. SPOP mutations occurred in only 4 patients (4\%) and, in keeping with previous reports, were mutually exclusive of ETS-gene fusions, present in $41 \%$ of patients.

Aberrations in genes involved in cell cycle regulation occurred in $7 \%$, due to copy-number alteration or mutation in CCND1, CDK4, CNKN1B, CDKN2A, and CDKN2B. Loss of $R B 1$ was observed less frequently $(1 \%)$ than reported in mCRPC (approximately 20\%). ${ }^{8}$ Aberrations in genes involved in chromatin remodeling occurred in $13 \%$, including mutation or copy-number alteration in KDM6A (4\%), mutation or rearrangement in KMT2C/MLL3 (3\%), or mutation in MLL2 (3\%). RAS/RAF/MEK pathway aberrations were present in $4 \%$, due to mutation or rearrangement of BRAF (3\%) or KRAS mutation (1\%). Mutations in the mismatch repair genes (MMR) MSH2 and MSH6 were observed in $3 \%$ overall, consistent with previous reports in mCRPC. ${ }^{7,11,12}$

The prevalence of DDR deficiency was determined in 185 patients through combining cohorts $1 \mathrm{a}$ and $1 \mathrm{~b}$. Aberrations in one or more of 14 genes involved in DDR were identified in $14 \%$. Two patients harbored pathogenic BRCA2 mutations, with 1 case shown to have 2 . The most frequently mutated genes involved in DDR were ATM $(8 \%)$ and CDK12 (5\%). Samples in which alterations in DDR genes were detected by Foundation Medicine were selected for concordance analysis, together with those with sufficient remaining DNA. Seventeen of 20 samples passed DNA quality control, and sequencing results performed by Almac Diagnostics were concordant in 16 of 17 . The only discordant result was ATM copy-number loss, because the Almac assay was not validated to detect copy-number alterations.

\section{Prospective Validation and Identification of Germline DDR Deficiency in mHSPC}

In stage 2, prospective screening validated the frequency of somatic alterations in the 14 specified genes involved in DDR, which were detected in $11 \%$. The most frequently mutated genes involved in DDR were again ATM and CDK12, present in $3 \%$ and $2 \%$, respectively. One patient had a CHEK2 mutation, 1 had an NBN mutation, and 1 had a RAD54L mutation. All positive results were externally 
TABLE 1. Patient Characteristics

\begin{tabular}{|c|c|c|c|c|c|c|}
\hline Baseline Characteristic & $\begin{array}{l}\text { Cohort } 1 \\
(n=185)\end{array}$ & $\begin{array}{c}\text { Comparative ITTa } \\
(n=1,744)\end{array}$ & $P^{b}$ & $\begin{array}{l}\text { Cohort } 2^{c} \\
(n=88)\end{array}$ & $\begin{array}{c}\text { Comparative } \text { ITT }^{\mathrm{a}} \\
(\mathrm{n}=204)\end{array}$ & $P^{b}$ \\
\hline \multicolumn{7}{|l|}{ Age at randomization, years } \\
\hline Median & 69 & 68 & & 68 & 68 & \\
\hline IQR & $63-75$ & $63-73$ & & $62-73$ & $63-75$ & \\
\hline \multicolumn{7}{|l|}{ Presenting PSA, ng/mL } \\
\hline Median & 93 & 96 & & 81 & 67 & \\
\hline IQR & 25-393 & 31-331 & & $22-250$ & $20-353$ & \\
\hline \multicolumn{7}{|l|}{ T stage } \\
\hline$\leq \mathrm{T} 2$ & $19(10)$ & $203(12)$ & \multirow[t]{4}{*}{.816} & $5(6)$ & $15(7)$ & \multirow[t]{4}{*}{.868} \\
\hline T3 & $102(55)$ & $989(57)$ & & $51(58)$ & $108(53)$ & \\
\hline T4 & $51(28)$ & $429(25)$ & & $23(26)$ & 59 (29) & \\
\hline Tx & $13(7)$ & $123(7)$ & & $9(10)$ & $22(11)$ & \\
\hline \multicolumn{7}{|l|}{ Nodal stage } \\
\hline NO & $58(31)$ & $581(33)$ & \multirow[t]{3}{*}{.863} & $22(25)$ & $57(28)$ & \multirow[t]{3}{*}{.103} \\
\hline N1 & $115(62)$ & $1,051(60)$ & & $66(75)$ & $138(68)$ & \\
\hline $\mathrm{Nx}$ & $12(6)$ & $112(6)$ & & $0(0)$ & $9(4)$ & \\
\hline \multicolumn{7}{|l|}{ Disease category } \\
\hline De novo M1 & $182(98)$ & $1,664(96)$ & \multirow[t]{2}{*}{.089} & $88(100)$ & $193(95)$ & \multirow[t]{2}{*}{-.061} \\
\hline Relapsed M1 & $3(2)$ & $80(4)$ & & $0(0)$ & $11(5)$ & \\
\hline \multicolumn{7}{|l|}{ Metastatic distribution } \\
\hline Bone only & $114(62)$ & $1,118(64)$ & \multirow[t]{3}{*}{.775} & $41(47)$ & $107(52)$ & \multirow[t]{3}{*}{.665} \\
\hline Distant node only & $20(11)$ & $185(11)$ & & $12(14)$ & $28(14)$ & \\
\hline Bone and nodal or other & $51(28)$ & $441(25)$ & & 34 (39) & $69(34)$ & \\
\hline \multicolumn{7}{|l|}{ Gleason } \\
\hline$\leq 7$ & $27(15)$ & 335 (19) & \multirow[t]{3}{*}{.311} & $13(15)$ & $31(15)$ & \multirow[t]{3}{*}{.768} \\
\hline $8-10$ & $149(81)$ & $1,329(76)$ & & $73(83)$ & $165(81)$ & \\
\hline Unknown & $9(5)$ & $80(5)$ & & $2(2)$ & $8(4)$ & \\
\hline \multicolumn{7}{|l|}{ Performance status } \\
\hline 0 & $131(71)$ & $1,242(71)$ & & $71(81)$ & $154(75)$ & \\
\hline 1 & $52(28)$ & $486(28)$ & & $16(18)$ & $47(23)$ & \\
\hline 2 & $2(1)$ & $16(1)$ & & $1(1)$ & $3(1)$ & \\
\hline
\end{tabular}

NOTE. Data are presented as No. (\%) unless otherwise noted. The trial protocol did not collect data on ethnicity or family history, and therefore this information is unknown.

Abbreviations: IQR, interquartile range; ITT, intention to treat; PSA, prostate-specific antigen.

${ }^{a}$ Comparative ITT defined as metastatic disease at trial entry, randomized during the same time period at the sites contributing samples to this analysis.

${ }^{\mathrm{b}} P$ values are from $\chi^{2}$ tests comparing the distribution of patients within each type of baseline characteristic between the cohort subgroups and the comparative ITT population. There is no compelling evidence that the cohort populations do not differ meaningfully from the comparative ITT population in terms of baseline characteristics.

'Baseline characteristics were collected at randomization, and therefore are unknown for the 11 participants who did not subsequently enter random.

validated using a clinically accredited assay (Foundation Medicine). Nine of 10 results were verified, with the exception of the RAD54L mutation, resulting in an estimate of DDR deficiency in stage 2 of $10 \%$. Results from germline DNA analysis were available for 89 of 93 patients (Fig 4). Overall, $5(5 \%)$ of 89 patients were found to harbor germline aberrations in genes involved in DDR; 2 harbored BRCA2 mutations, 2 ATM mutations, and 1 a CHEK2 mutation. No germline mutations were identified in BARD1, BRCA1, BRIP1, PALB2, RAD51C, or RAD51D or other genes involved in mismatch repair ( $M L H 1, M S H 2, M S H 3$ ). See Appendix Table A1 for a full list of genes covered by each assay. 


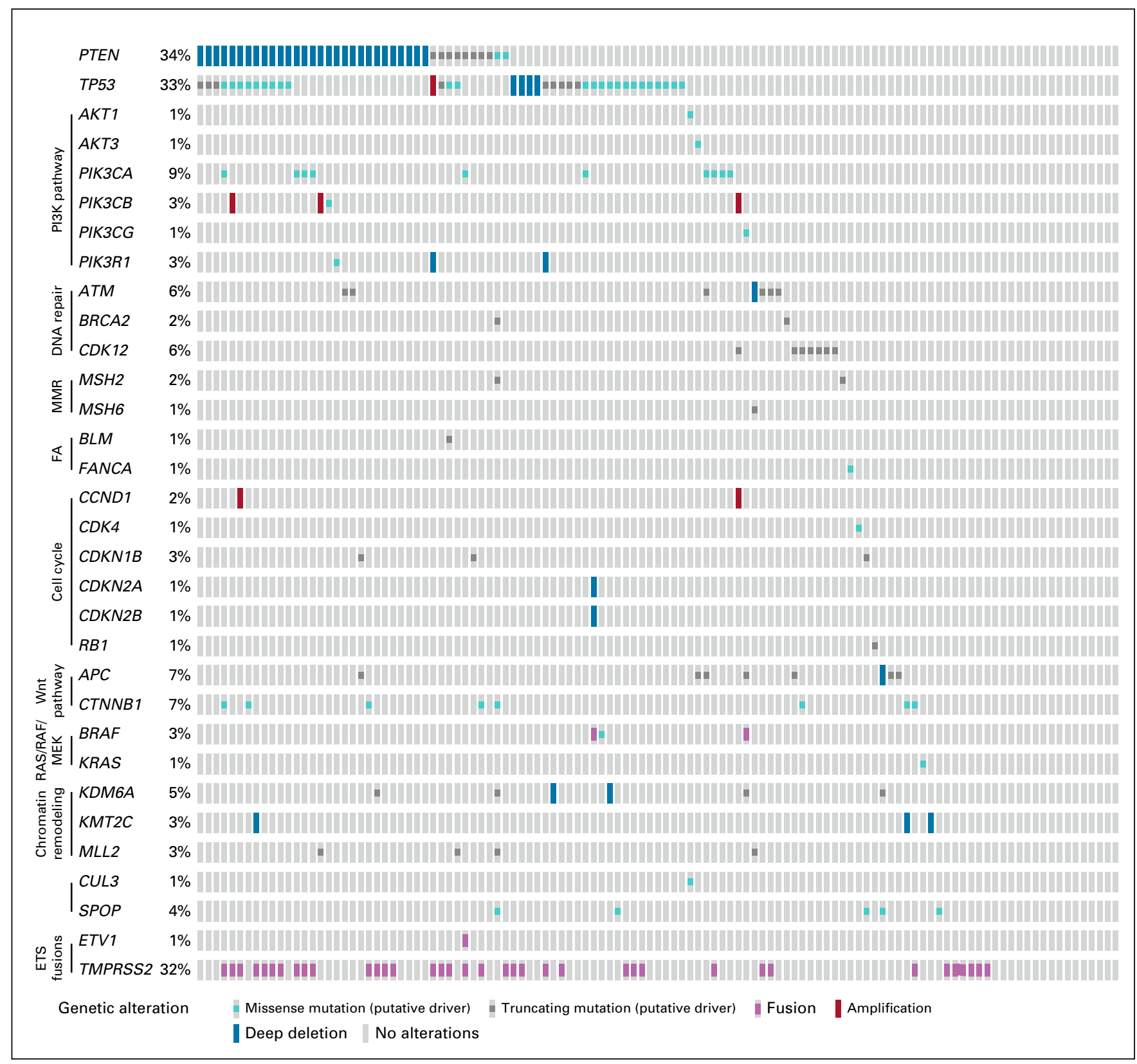

FIG 2. Selected genomic aberrations identified in cohort 1a $(n=115)$. Samples were sequenced by Foundation Medicine with targeted next-generation sequencing assay (T7). Thirty-nine genes of interest were defined based on prior publications ${ }^{7,12}$; those 32 found to be aberrant are presented here. See Appendix Table A1 for gene lists. The data are publicly available via cBioPortal.

\section{Genomic Characterization According to Metastatic Burden}

Metastatic burden was known for 94 (51\%) of 185 of cohort 1 (Fig 1). Fifty-four patients (57\%) had highvolume metastatic disease, and 40 (43\%) had lowvolume disease, consistent with previous reports (see Appendix Table A3, online only for comparative baseline characteristics). ${ }^{25}$ Sixty-three of 94 patients in whom metastatic burden was known were in cohort $1 a$, sequenced by Foundation Medicine. In an exploratory analysis in this small subset, differences according to metastatic burden were observed. Overall DDR deficiency was detected in 18 (19\%) of 94 , and we observed a trend for a higher prevalence in high-volume disease: $22 \%$, compared with $15 \%$ in low-volume disease. The only BRCA2 mutant case and both MMR-deficient cases had high-volume disease. High-volume disease was associated with a higher frequency of aberrations in Wnt signaling (16\% $v 4 \%$ ), and alterations in genes involved in chromatin remodeling (16\% v 8\%; Fig 5). In contrast, aberrations in PTEN and the PI3K pathway were equally prevalent. TP53 and ETS fusions were both more frequent 


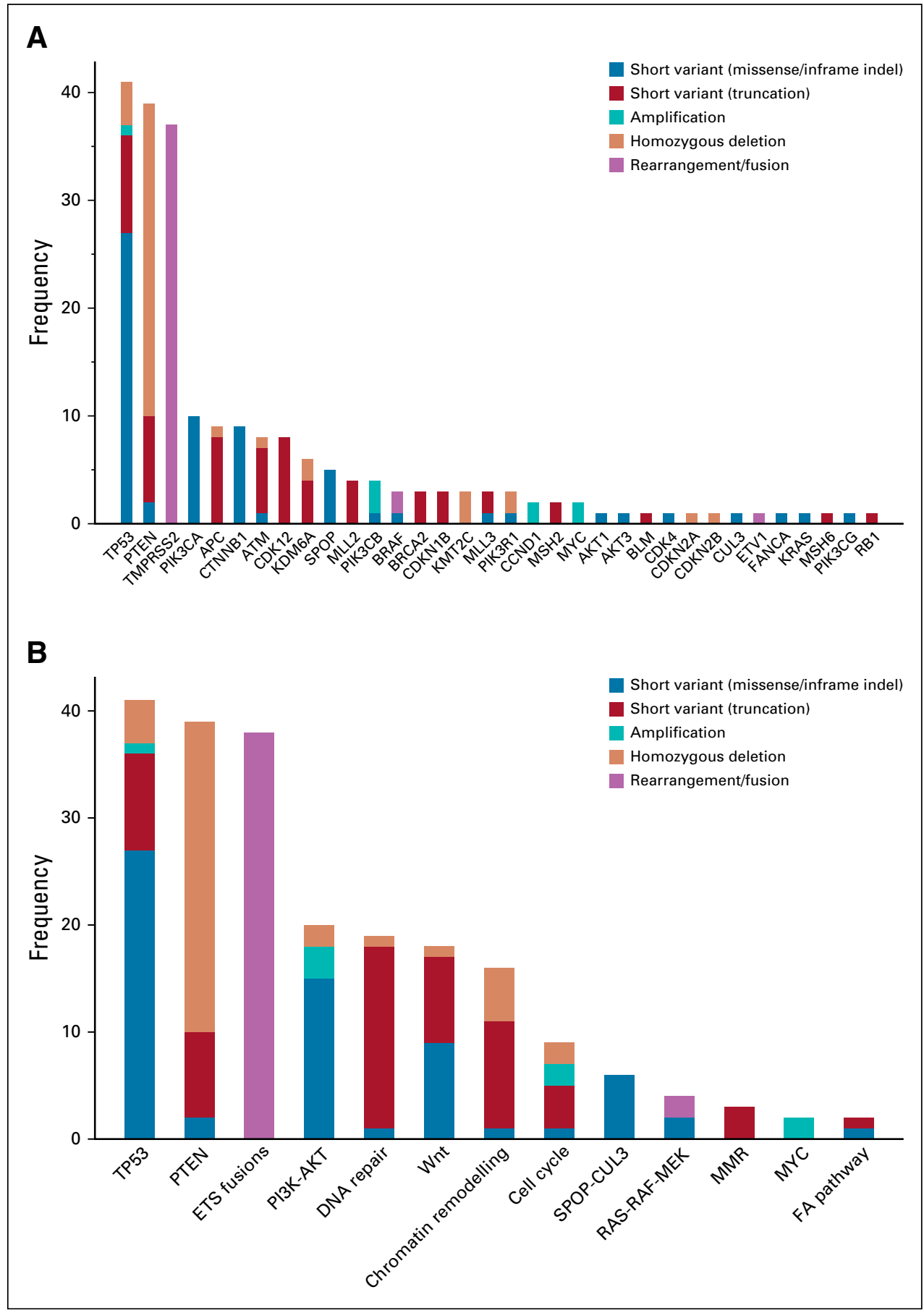

FIG 3. (A) Frequency of aberration by gene in cohort 1a. (B) Frequency of pathway aberrations in cohort 1 a.

in low-volume disease $(31 \% \vee 21 \%$ and $38 \% \vee 19 \%$, respectively). We did not find evidence for statistically significant differences in the frequency of specific mutations between high- and low-volume subgroups (Fisher's exact test: $P>$.05).

\section{DISCUSSION}

We demonstrate that it is feasible to use residual diagnostic FFPE tumor biopsy specimens to perform tNGS.
Our results suggest that the time between obtaining the biopsy and sequencing influences the sequencing success rate, as this was the main difference between cohort 2 and cohort 1 (92\% and $65 \%$ respectively). In cohort 1 , archival samples were up to 7 years old, whereas in cohort 2 , all were $<8$ months. Other differences included biopsy date (before April 2017 for cohort 1, December 2017August 2018 for cohort 2), but we did not identify any change in sample processing protocols, and most sites 


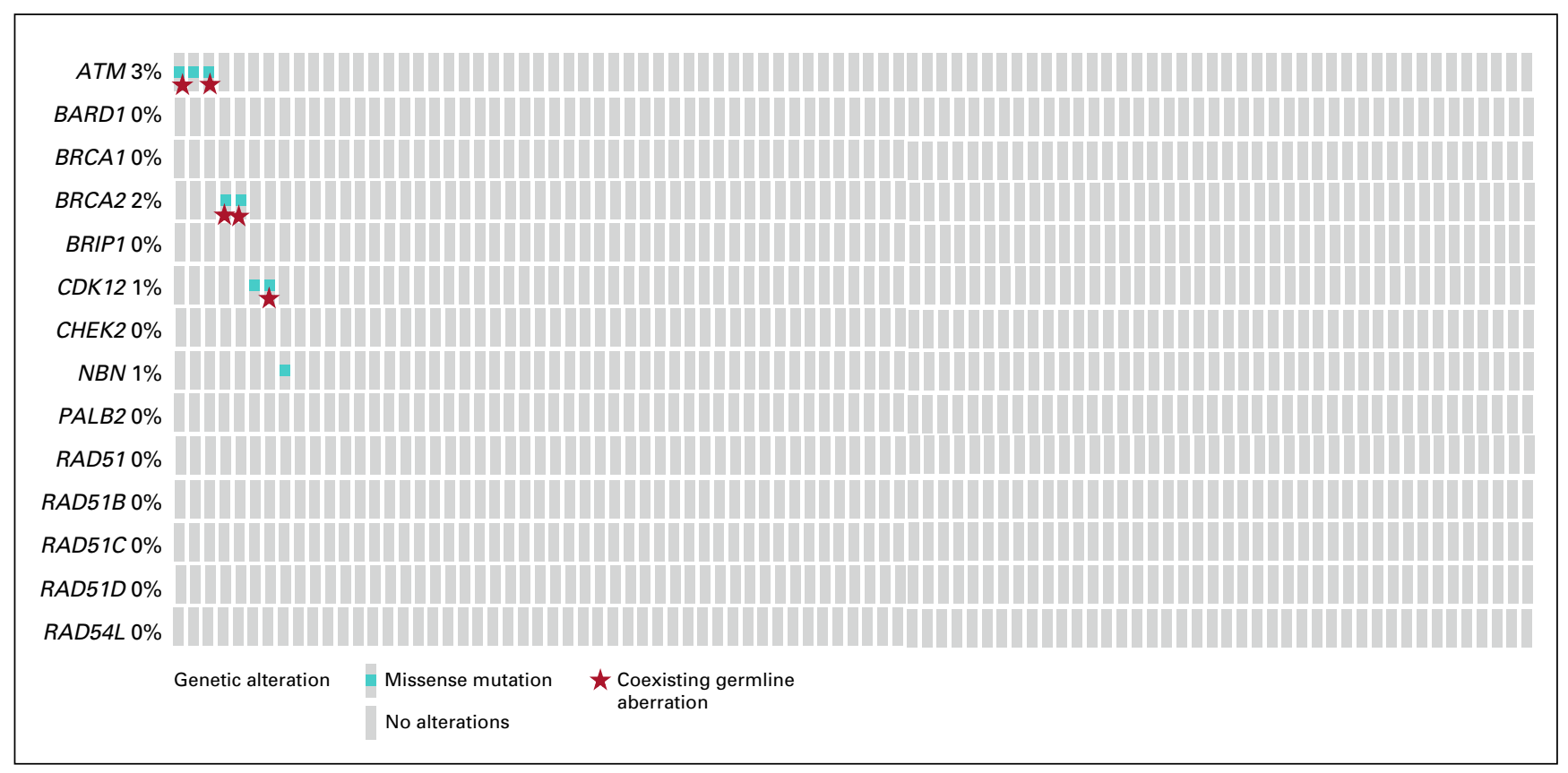

FIG 4. Prevalence of somatic and germline DNA damage repair (DDR) in cohort 2. Three genes were only included in tumor sequencing panel (ie, 11 genes were evaluated in both tumor and germline DNA). In 5 cases where a somatic change was detected in one gene involved in DDR, a germline change was also reported. However, because of differences in annotation, it is uncertain as to whether these all represent second-hit somatic mutations or coexisting mutations within the same gene. The prevalence of germline DDR mutations is lower than somatic mutations, suggesting that a screening strategy that focuses solely on germline aberrations will identify a smaller population.

contributed to both cohorts. When compared with published data, our results suggest the genomic profile of de novo metastatic prostate cancer is different from localized prostatectomy cohorts and, notably, shares similarities with heavily pretreated $\mathrm{mCRPC}$, other than for the absence of $A R$ aberrations and low prevalence of $R B 1$ loss. ${ }^{11,12,16}$ We identified a higher prevalence of PI3K/AKT pathway aberrations compared with the MSK-IMPACT mHSPC population, in which half had relapsed metastatic disease after previous treatment of localized $\mathrm{PCa}{ }^{7}$ Differences in genomic profiles of de novo compared with relapsed metastatic disease may explain the recently proposed difference in benefit with docetaxel for these 2 clinical presentations. ${ }^{24} A R$ aberrations were detected in the MSK-IMPACT mHSPC cohort but were limited to those with prior ADT exposure, suggesting the development of subclinical mCRPC, and were not observed in our cohort, who were all treatment naive. We observed a lower prevalence of SPOP mutations (4\%) compared with published estimates in prostatectomy series $(11 \%-13 \%)$ but similar to mCRPC (approximately 5\%). ${ }^{12,15,27}$ This finding is consistent with data from cohorts enriched for SPOP mutations, where the lowest frequency was observed in de novo metastatic disease. ${ }^{9,28}$ The high prevalence observed in localized cohorts suggests it is a feature of good-prognosis disease. ${ }^{28}$

In an exploratory analysis, high- and low-metastatic burden tumors had a similar prevalence of aberrations in the
PI3K/AKT pathway, but aberrations involving MMR (5\% $v 0 \%$ ) and DDR (22\% v 15\%), Wnt (16\% v 4\%), and chromatin remodeling (16\% $\vee 8 \%)$ pathways appeared more common in high-volume disease, with a prevalence similar to mCRPC. Validation in a larger cohort is required, but this suggests biologic differences may underpin this classification. The prevalence of somatic BRCA1 and $B R C A 2$ mutations was lower than reported in other mHSPC cohorts and heavily pretreated mCRPC. ${ }^{11,12,16}$ Consistent with this, we observed a lower frequency of germline DDR aberrations. This may be a result of differences in patient selection and population: the STAMPEDE trial recruits at both rural general hospitals and tertiary referral centers. This contrasts with other cohorts conducted primarily at tertiary referral centers. ${ }^{26,29}$

The sample size for this analysis offers limited power to compare the frequency of specific mutations between high- and low-volume subgroups. However, the results are nonetheless interesting, given prior studies suggested worse outcome for cancers harboring aberrations in DDR or the WNT pathway that appeared to occur more commonly in high-volume disease. This contrasts with ETS gene fusions that appear to occur at a higher frequency in lowvolume disease and have previously been associated with no difference in outcome. Also, the similar prevalence of a number of aberrations suggests that differences observed were not a result of lower tumor purity in low-volume cancer samples. 


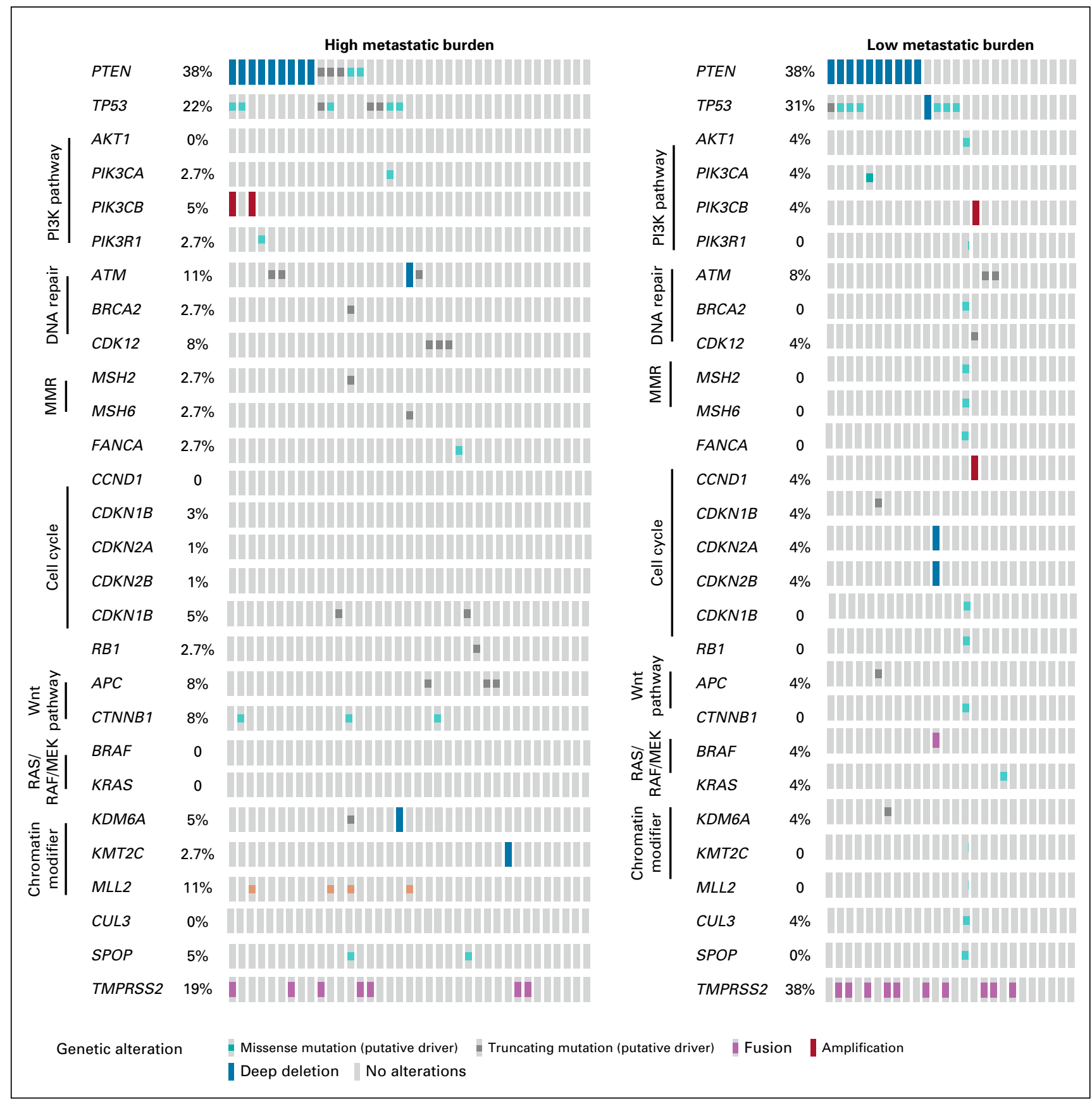

FIG 5. Comparative genomic profile in high- and low-volume metastatic disease $(n=64)$. Samples were sequenced by Foundation Medicine targeted next-generation sequencing assay (T7). Thirty-nine genes of interest were defined based on prior publications ${ }^{7,12}$; those 28 found to be aberrant are presented here. See Appendix Table A1 for gene lists.

The size of our cohort does not allow definitive assessment of less-prevalent subgroups, such as BRCA mutants, but as these patients have the best responses to PARP inhibition, this finding may have implications for trial designs evaluating this strategy in this setting. ${ }^{30}$ Wnt signaling modulates cell growth and survival predominately via maintaining stem cells in a selfrenewing state. ${ }^{31}$ Androgen-modulating therapies have been shown to modify Wnt signaling, and Wnt pathway aberrations were associated with reduced benefit from AR-targeted therapies, suggesting this finding may also have therapeutic relevance. ${ }^{32}$ The prevalence of MMR deficiency was relatively low and consistent with prior mCRPC data. 8,12,27,33

A limitation of the current work is that this study was performed in participants of an ongoing trial, randomly assigned to receive different therapies. Second, the sequencing 
technique used may miss, and therefore underestimate, the frequency of MMR gene aberrations secondary to complex structural rearrangements involving noncoding regions and larger indels that account for half of hypermutated mCRPC. ${ }^{26,34}$ Nonetheless, comparisons between sequencing approaches can be challenging, and so the reliability of the estimate of DDR deficiency is strengthened by the use of 2 tNGS assays, shown to have good concordance. Finally, the trial does not routinely collect ethnicity, which may limit applicability to other populations, especially in terms of germline risk.

\section{AFFILIATIONS}

${ }^{1}$ MRC Clinical Trials Unit at UCL, Institute of Clinical Trials and Methodology, University College London, London, United Kingdom ${ }^{2}$ University College London Cancer Institute, London, United Kingdom ${ }^{3} \mathrm{GU}$ Research and FASTMAN groups, Manchester Cancer Institute, University of Manchester, Manchester, United Kingdom

${ }^{4}$ Department of Surgery, The Christie NHS Foundation Trust, Manchester and Salford Royal NHS Foundation Trusts, Manchester, United Kingdom ${ }^{5}$ Cardiff University, Cardiff, United Kingdom

${ }^{6}$ Clatterbridge Cancer Centre, Liverpool, United Kingdom

${ }^{7}$ Clovis Oncology, Boulder, CO

${ }^{8}$ The Royal Marsden NHS Foundation Trust, London, United Kingdom

${ }^{9}$ Institute of Cancer Research, London, United Kingdom

${ }^{10}$ Guys and St Thomas' Hospitals, London, United Kingdom

STAMPEDE Investigators from sub-study sites: Jason Lester, Delia Pudney, John Logue, Olivera Frim, Robert Brierly, Abdel Hamid, Uschi Hofmann, Denise Sheehan, Anna Lydon, Amit Bahl, Thiagarajan Sreenivasan, Neil McPhail, Joji Joseph, Omi Parikh, Simon Chowdhury, Ursula McGovern, Mohammed Kagki, Robert Hughes, Anna Tran, Tony Elliot, John Graham, Sue Brock, Carmel Pezaro, Georgina Walker, Nishi Gupta, Joanne Gale, Imtiaz Ahmed, Ashok Nikapota, Dakshinamoorthy Muthukumar, Warren Grant, Clive Peedell, Simon Crabb, Tim Porter. MRC CTU at UCL: Dominic Hague, Carly Au, Francesca Schiavone, Orla Prendivill, Mazna Anjum, Nour Merzouk. Shabinah Ali. Wales Cancer Bank: Alison Parry-Jones, David Griffiths, Malcolm Mason, Fiona Morgan. Almac Diagnostics: Richard Kennedy, Laura Nelson. Foundation Medicine: Murtaza Mehdi, Jon Chung. Color Genomics: Patrick Miller

\section{CORRESPONDING AUTHOR}

Gerhardt Attard, MD, PhD, University College London Cancer Institute, Paul O'Gorman Building, 72 Huntley St, London, N/A WC1E 6DD, United Kingdom; e-mail: g.attard@ucl.ac.uk.

\section{PRIOR PRESENTATION}

Presented at the ASCO Annual Meeting, Chicago, IL, May 31-June 4, 2019.

\section{CLINICAL TRIAL INFORMATION NCT00268476}

\section{AUTHOR CONTRIBUTIONS}

Conception and design: Clare Gilson, Duncan C. Gilbert, Noel W. Clarke, Chris Wanstall, Malcolm D. Mason, Zafar Malik, Nicholas D. James, Ruth E. Langley, Matthew R. Sydes, Gerhardt Attard, Simon Chowdhury Administrative support: Nafisah B. Atako

Provision of study material or patients: Nafisah B. Atako, Nicholas D. James, Matthew R. Sydes
In summary, we present baseline genomic data obtained from a representative subset of a large clinical trial in $\mathrm{mHSPC}$. We demonstrate that it is feasible to implement prospective genomic characterization and report a high sequencing success using residual diagnostic biopsy FFPE samples. We demonstrate that a specific genomic profile is observed in de novo $\mathrm{mHSPC}$, and these prevalence data will inform trial design in this setting. Adaptive protocols like STAMPEDE provide an important opportunity to incorporate genomic or molecular characterization with the aim of rational treatment selection.

Collection and assembly of data: Clare Gilson, Nafisah B. Atako, Adnan Ali, Alex Hoyle, Noel W. Clarke, Chris Wanstall, Christopher Brawley, Malcolm D. Mason, Zafar Malik, Andrea Loehr, Alison Parry-Jones, Rosalind Eeles, Matthew R. Sydes, Gerhardt Attard, Simon Chowdhury

Data analysis and interpretation: Clare Gilson, Fiona Ingleby, Marina A. Parry, Noel W. Clarke, Melissa Gannon, Malcolm D. Mason, Zafar Malik, Andrew Simmons, Andrea Loehr, Zsofia Kote-Jarai, Nicholas D. James, Claire Amos, Mahesh K. B. Parmar, Matthew R. Sydes, Gerhardt Attard, Simon Chowdhury

Manuscript writing: All authors

Final approval of manuscript: All authors

Accountable for all aspects of the work: All authors

\section{AUTHORS' DISCLOSURES OF POTENTIAL CONFLICTS OF INTEREST}

The following represents disclosure information provided by authors of this manuscript. All relationships are considered compensated unless otherwise noted. Relationships are self-held unless noted. I = Immediate Family Member, Inst = My Institution. Relationships may not relate to the subject matter of this manuscript. For more information about ASCO's conflict of interest policy, please refer to www.asco.org/rwc or ascopubs. org/po/author-center.

Open Payments is a public database containing information reported by companies about payments made to US-licensed physicians (Open

Payments).

\section{Clare Gilson}

Research Funding: Clovis Oncology (Inst)

\section{Noel W. Clarke}

Honoraria: Janssen-Cilag, Astellas Pharma, AstraZeneca

Speakers' Bureau: AstraZeneca, Astellas Pharma, Bayer, Ferring, Ipsen, Sanofi, Amgen, Janssen

Research Funding: AstraZeneca (Inst)

Travel, Accommodations, Expenses: Ipsen, Janssen, Sanofi

Other Relationship: Astellas Pharma

\section{Christopher Brawley}

Research Funding: Astellas Pharma (Inst), Clovis Oncology (Inst), Janssen (Inst), Sanofi/Aventis (Inst), Pfizer (Inst), Novartis (Inst)

\section{Malcolm D. Mason}

Other Relationship: Endocyte, Clovis

Zafar Malik

Consulting or Advisory Role: Sanofi/Aventis

\section{Andrew Simmons}

Employment: Clovis Oncology

Stock and Other Ownership Interests: Clovis Oncology

Consulting or Advisory Role: Redwood Bioscience 


\section{Andrea Loehr}

Employment: Clovis Oncology

Stock and Other Ownership Interests: Clovis Oncology

Rosiland Eeles

Honoraria: Janssen-Cilag

Speakers' Bureau: Janssen-Cilag, University of Chicago, GU-ASCO

meeting in San Francisco (Speaker)

\section{Nicholas D. James}

Honoraria: Sanofi, Bayer, Janssen, Astellas Pharma

Consulting or Advisory Role: Sanofi, Bayer, Astellas Pharma, Janssen, Clovis, EUSA Pharma, Pfizer

Speakers' Bureau: Pierre Fabre, Ferring, Sanofi, Astellas Pharma, Janssen Oncology, Merck

Research Funding: Janssen (Inst), Astellas Pharma (Inst), Pfizer (Inst), Sanofi (Inst), Novartis (Inst), AstraZeneca (Inst)

Travel, Accommodations, Expenses: Sanofi, Janssen

Ruth E. Langley

Consulting or Advisory Role: Bayer Schering Pharma (Inst)

Research Funding: Bayer Schering Pharma (Inst)

Matthew R. Sydes

Honoraria: Lilly, Sanofi, Janssen

Research Funding: Astellas Pharma, Janssen-Cilag, Pfizer, Novartis, Sanofi, Clovis Oncology

\section{Gerhardt Attard}

Honoraria: Janssen, Astellas Pharma, Janssen (I)

Consulting or Advisory Role: Janssen-Cilag, Veridex, Ventana Medical Systems, Astellas Pharma, Medivation, Novartis, Millennium, Abbott Laboratories, ESSA Pharma, Bayer, Pfizer, AstraZeneca, Ferring

Speakers' Bureau: Janssen, Astellas Pharma, Takeda, Sanofi, Ventana Medical Systems, Ipsen, AstraZeneca, Ferring

Research Funding: Janssen (Inst), Arno Therapeutics (Inst), Innocrin Pharmaceuticals (Inst)

Patents, Royalties, Other Intellectual Property: I am on the Institute of Cancer Research rewards to inventors list of abiraterone acetate.

Travel, Accommodations, Expenses: Janssen, Astellas Pharma, Medivation, Ventana Medical Systems, Abbott Laboratories, Bayer, ESSA Pharma, Janssen (I), Astellas Pharma (I), Pfizer, Ferring

Other Relationship: Institute of Cancer Research

\section{Simon Chowdhury}

Honoraria: Clovis Oncology, Novartis

Consulting or Advisory Role: Clovis Oncology, Astellas Pharma, Bayer, Pfizer, Janssen-Cilag

Speakers' Bureau: Pfizer

Research Funding: Sanofi/Aventis (Inst)

No other potential conflicts of interest were reported

\section{REFERENCES}

1. James ND, Spears MR, Clarke NW, et al: Survival with newly diagnosed metastatic prostate cancer in the "docetaxel Era": Data from 917 patients in the control arm of the STAMPEDE trial (MRC PR08, CRUK/06/019). Eur Urol 67:1028-1038, 2015

2. Chi KN, Agarwal N, Bjartell A, et al: First results from TITAN: A phase III double-blind, randomized study of apalutamide (APA) versus placebo (PBO) in patients (pts) with metastatic castration-sensitive prostate cancer (mCSPC) receiving androgen deprivation therapy (ADT). J Clin Oncol 37, 2019 (15_suppl; abstr 5006)

3. James ND, de Bono JS, Spears MR, et al: Abiraterone for prostate cancer not previously treated with hormone therapy. N Engl J Med 377:338-351, 2017

4. Fizazi K, Tran N, Fein L, et al: Abiraterone plus prednisone in metastatic, castration-sensitive prostate cancer. N Engl J Med 377:352-360, 2017

5. Sweeney CJ, Chen Y-H, Carducci M, et al: Chemohormonal therapy in metastatic hormone-sensitive prostate cancer. N Engl J Med 373:737-746, 2015

6. James ND, Sydes MR, Clarke NW, et al: Addition of docetaxel, zoledronic acid, or both to first-line long-term hormone therapy in prostate cancer (STAMPEDE): Survival results from an adaptive, multiarm, multistage, platform randomised controlled trial. Lancet 387:1163-1177, 2016

7. Abida W, Armenia J, Gopalan A, et al: Prospective genomic profiling of prostate cancer across disease states reveals germline and somatic alterations that may affect clinical decision making. JCO Precis Oncol 10.1200/PO.17.00029

8. Beltran H, Yelensky R, Frampton GM, et al: Targeted next-generation sequencing of advanced prostate cancer identifies potential therapeutic targets and disease heterogeneity. Eur Urol 63:920-926, 2013

9. Taylor BS, Schultz N, Hieronymus H, et al: Integrative genomic profiling of human prostate cancer. Cancer Cell 18:11-22, 2010

10. Cancer Genome Atlas Research Network: The molecular taxonomy of primary prostate cancer. Cell 163:1011-1025, 2015

11. Grasso CS, Wu YM, Robinson DR, et al: The mutational landscape of lethal castration-resistant prostate cancer. Nature 487:239-243, 2012

12. Robinson D, Van Allen EM, Wu YM, et al: Integrative clinical genomics of advanced prostate cancer. Cell 161:1215-1228, 2015 [Erratum: Cell 162:454, 2015 ]

13. Mateo J, Carreira S, Sandhu S, et al: DNA-repair defects and olaparib in metastatic prostate cancer. N Engl J Med 373:1697-1708, 2015

14. Baca SC, Prandi D, Lawrence MS, et al: Punctuated evolution of prostate cancer genomes. Cell 153:666-677, 2013

15. Barbieri CE, Baca SC, Lawrence MS, et al: Exome sequencing identifies recurrent SPOP, FOXA1 and MED12 mutations in prostate cancer. Nat Genet 44:685-689, 2012

16. Kumar A, Coleman I, Morrissey C, et al: Substantial interindividual and limited intraindividual genomic diversity among tumors from men with metastatic prostate cancer. Nat Med 22:369-378, 2016

17. STAMPEDE Trial: www.stampedetrial.org

18. Frampton GM, Fichtenholtz A, Otto GA, et al: Development and validation of a clinical cancer genomic profiling test based on massively parallel DNA sequencing. Nat Biotechnol 31:1023-1031, 2013

19. Chou D, Chen X, Purdy A, et al: Abstract 3732: Analytical performance of TruSight Tumor 170 on small nucleotide variations and gene amplifications using DNA from formalin-fixed, paraffin-embedded (FFPE) solid tumor samples. Cancer Res 77:3732, 2017

20. LoCoco JS, Teng L, Chou D, et al: Abstract 5354: Evaluation of quantity, quality and performance with the TruSight Tumor 170 solid tumor profiling assay of nucleic acids extracted from formalin-fixed paraffin-embedded (FFPE) tissue sections. Cancer Res 77:5354, 2017

21. Swisher EM, Lin KK, Oza AM, et al: Rucaparib in relapsed, platinum-sensitive high-grade ovarian carcinoma (ARIEL2 Part 1): An international, multicentre, open-label, phase 2 trial. Lancet Oncol 18:75-87, 2017

22. Neben CL, Zimmer AD, Stedden W, et al: Multi-gene panel testing of 23,179 individuals for hereditary cancer risk identifies pathogenic variant carriers missed by current genetic testing guidelines. J Mol Diagn 21:646-657, 2019

23. Hoyle AP, Ali A, James ND, et al: Abiraterone in "high-" and "low-risk" metastatic hormone-sensitive prostate cancer. Eur Urol 76:719-728, 2019 
24. Clarke NW, Ali A, Ingleby FC, et al: Addition of docetaxel to hormonal therapy in low- and high-burden metastatic hormone sensitive prostate cancer: Long-term survival results from the STAMPEDE trial. Ann Oncol 30:1992-2003, 2019

25. Parker CC, James ND, Brawley CD, et al: Radiotherapy to the primary tumour for newly diagnosed, metastatic prostate cancer (STAMPEDE): A randomised controlled phase 3 trial. Lancet 392:2353-2366, 2018

26. Pritchard CC, Mateo J, Walsh MF, et al: Inherited DNA-repair gene mutations in men with metastatic prostate cancer. N Engl J Med 375:443-453, 2016

27. Armenia J, Wankowicz SAM, Liu D, et al: The long tail of oncogenic drivers in prostate cancer. Nat Genet 50:645-651, 2018 [Erratum: Nat Genet 51:1194, 2019]

28. Boysen G, Rodrigues DN, Rescigno P, et al: SPOP-mutated/CHD1-deleted lethal prostate cancer and abiraterone sensitivity. Clin Cancer Res 24:5585-5593, 2018

29. Abida W, Armenia J, Gopalan A, et al: Prospective genomic profiling of prostate cancer across disease states reveals germline and somatic alterations that may affect clinical decision making. JCO Precis Oncol 10.1200/PO.17.00029

30. Marshall $\mathrm{CH}$, Sokolova AO, McNatty AL, et al: Differential response to olaparib treatment among men with metastatic castration-resistant prostate cancer harboring BRCA1 or BRCA2 versus ATM mutations. Eur Urol 76:452-458, 2019

31. Nusse R: Wnt signaling and stem cell control. Cell Res 18:523-527, 2008

32. Wang L, Dehm SM, Hillman DW, et al: A prospective genome-wide study of prostate cancer metastases reveals association of WNT pathway activation and increased cell cycle proliferation with primary resistance to abiraterone acetate-prednisone. Ann Oncol 29:352-360, 2018

33. Chung JH, Dewal N, Sokol E, et al: Prospective comprehensive genomic profiling of primary and metastatic prostate tumors. JCO Precis Oncol $10.1200 /$ P0.18.00283

34. Pritchard CC, Morrissey C, Kumar A, et al: Complex MSH2 and MSH6 mutations in hypermutated microsatellite unstable advanced prostate cancer. Nat Commun 5:4988, 2014 
APPENDIX

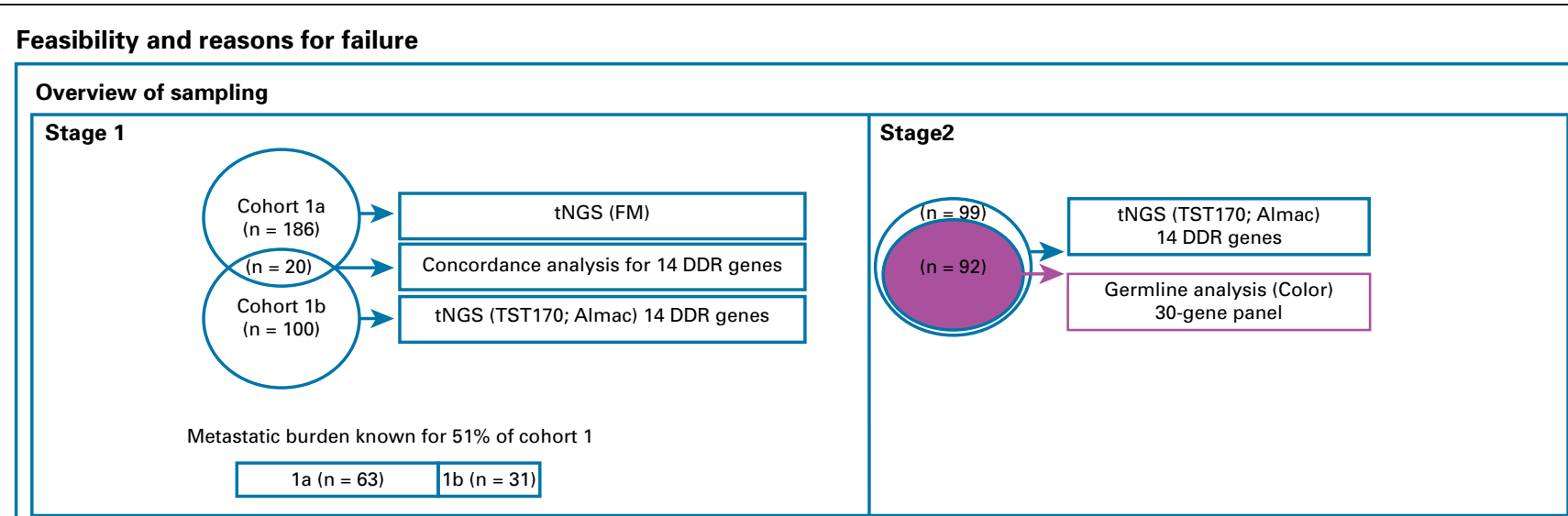

Feasibility data including reasons for sequencing failure

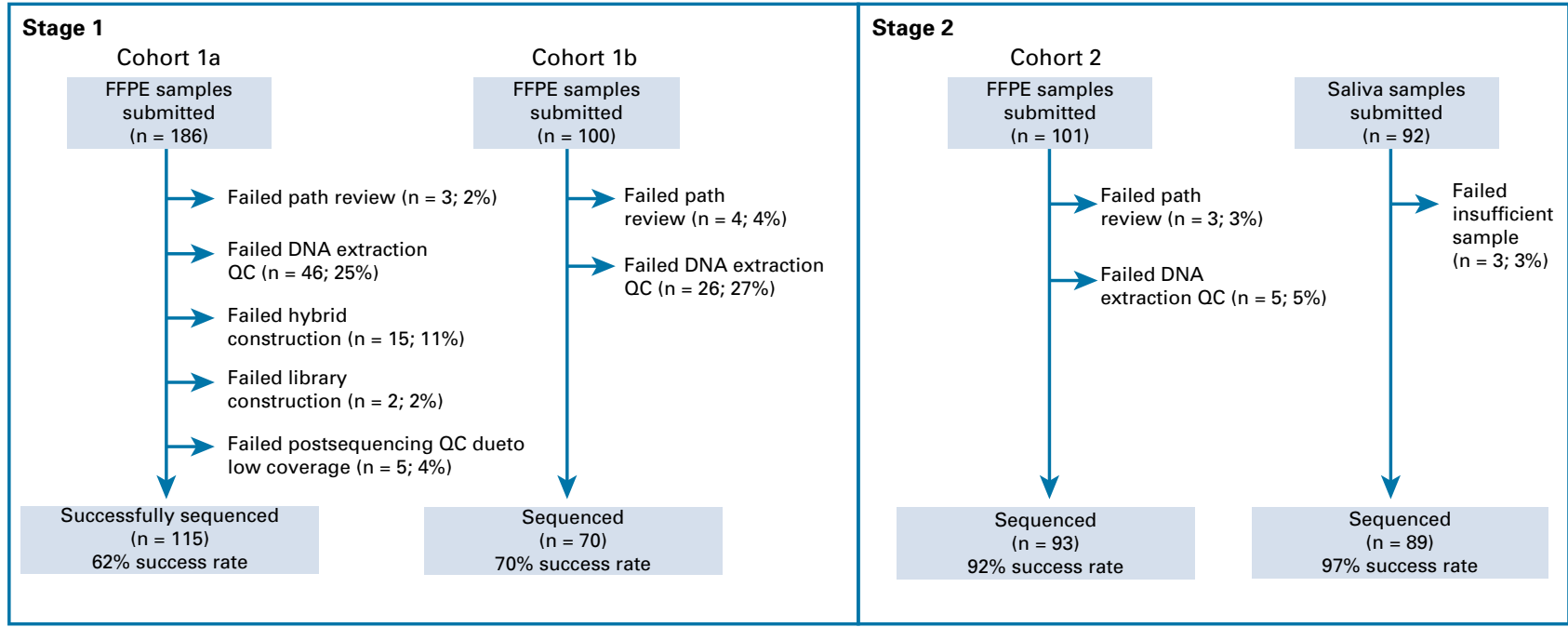

FIG A1. Feasibility and reasons for failure. DDR, DNA damage repair; FFPE, formalin-fixed, paraffin-embedded; FM, Foundation Medicine; QC, quality control; tNGS, targeted next-generation sequencing. 
TABLE A1. Gene List for Each Assay

\begin{tabular}{|c|c|c|}
\hline FM Genes of Interest & $\begin{array}{c}\text { Almac DDR } \\
14 \text { Gene Panel }\end{array}$ & $\begin{array}{c}\text { Color } \\
\text { Germline Panel }\end{array}$ \\
\hline AKT1 & ATM & $A P C$ \\
\hline AKT3 & BARD1 & ATM \\
\hline$A P C$ & $B R C A 1$ & $B A P 1$ \\
\hline ATM & BRCA2 & $B A R D 1$ \\
\hline$B L M$ & $B R I P 1$ & BMPR1A \\
\hline$B R A F$ & CDK12 & $B R C A 1$ \\
\hline BRCA2 & CHEK2 & BRCA2 \\
\hline CCND1 & $N B N$ & $B R I P 1$ \\
\hline$C D K 1$ & PALB2 & $\mathrm{CDH} 1$ \\
\hline CDK4 & RAD51 & CDK4 \\
\hline CDKN1B & RAD51B & CDKN2A \\
\hline CDKN2A & RAD51C & CHEK2 \\
\hline$C D K N 2 B$ & RAD51D & EPCAM \\
\hline CTNNB1 & RAD54L & GREM1 \\
\hline CUL3 & & MITF \\
\hline ETV1 & & MLH1 \\
\hline FANCA & & $\mathrm{MSH} 2$ \\
\hline$K D M 6 A$ & & MSH6 \\
\hline KMT2C & & MUTYH \\
\hline KRAS & & $N B N$ \\
\hline MLL2 & & PALB2 \\
\hline $\mathrm{MSH} 2$ & & PMS2 \\
\hline MSH6 & & POLD1 \\
\hline PIKЗCA & & POLE \\
\hline PIKЗCB & & PTEN \\
\hline PIK3CG & & RAD51C \\
\hline PIK3R1 & & RAD51D \\
\hline PTEN & & SMAD4 \\
\hline$R B 1$ & & STK11 \\
\hline SPOP & & TP53 \\
\hline TMPRSS2 & & \\
\hline
\end{tabular}

Abbreviation: FM, Foundation Medicine. 
TABLE A2. Comparative baseline characteristics according to sequencing success

\begin{tabular}{|c|c|c|c|c|c|}
\hline Baseline Characteristic & $\begin{array}{c}\text { All } \\
(\mathrm{N}=286)\end{array}$ & $\begin{array}{l}\text { Sequenced } \\
(n=185)\end{array}$ & $P^{a}$ & $\begin{array}{l}\text { Sequencing Failed } \\
(n=101)\end{array}$ & $P^{a}$ \\
\hline \multicolumn{6}{|l|}{ Age at randomization, years } \\
\hline Median & 68 & 69 & & 67 & \\
\hline IQR & $63-73$ & $63-75$ & & $62-71$ & \\
\hline \multicolumn{6}{|l|}{ Presenting PSA, ng/mL } \\
\hline Median & 127 & 93 & & 149 & \\
\hline IQR & $35-400$ & $25-393$ & & $51-435$ & \\
\hline \multicolumn{6}{|l|}{ T stage } \\
\hline$\leq \mathrm{T} 2$ & $27(9)$ & $19(10)$ & \multirow[t]{4}{*}{.975} & $8(8)$ & \multirow[t]{4}{*}{.915} \\
\hline T3 & $162(57)$ & $102(55)$ & & $60(59)$ & \\
\hline T4 & 79 (28) & $51(28)$ & & $28(28)$ & \\
\hline Tx & $18(6)$ & $13(7)$ & & $5(5)$ & \\
\hline \multicolumn{6}{|l|}{ Nodal state } \\
\hline NO & $103(36)$ & $58(31)$ & \multirow[t]{3}{*}{.5601} & $45(45)$ & \multirow[t]{3}{*}{.288} \\
\hline N1 & $164(57)$ & $115(62)$ & & $49(48)$ & \\
\hline $\mathrm{Nx}$ & $19(7)$ & $12(6)$ & & $7(7)$ & \\
\hline \multicolumn{6}{|l|}{ Disease category } \\
\hline De novo $\mathrm{M} 1$ & 282 (99) & $182(98)$ & \multirow[t]{2}{*}{.998} & $100(99)$ & \multirow[t]{2}{*}{.996} \\
\hline Relapsed M1 & $4(1)$ & $3(2)$ & & $1(1)$ & \\
\hline \multicolumn{6}{|l|}{ Metastatic distribution } \\
\hline Bone only & $191(67)$ & $114(62)$ & \multirow[t]{3}{*}{.519} & 77 (76) & \multirow[t]{3}{*}{.208} \\
\hline Distant node only & $27(9)$ & $20(11)$ & & $7(7)$ & \\
\hline Bone and nodal or other & $68(24)$ & $51(28)$ & & $17(17)$ & \\
\hline \multicolumn{6}{|l|}{ Gleason } \\
\hline$\leq 7$ & $50(17)$ & $27(15)$ & \multirow[t]{3}{*}{.707} & $23(23)$ & \multirow[t]{3}{*}{.500} \\
\hline $8-10$ & $222(78)$ & $149(81)$ & & $73(72)$ & \\
\hline Unknown & $14(5)$ & $9(5)$ & & $5(5)$ & \\
\hline \multicolumn{6}{|l|}{ Performance status } \\
\hline 0 & $208(73)$ & $131(71)$ & \multirow[t]{3}{*}{.837} & 77 (76) & \multirow[t]{3}{*}{.673} \\
\hline 1 & $74(26)$ & $52(28)$ & & $22(22)$ & \\
\hline 2 & $4(1)$ & $2(1)$ & & $2(2)$ & \\
\hline
\end{tabular}

NOTE. Data are presented as No. (\%) unless otherwise noted.

Abbreviations: IQR, interquartile range; PSA, prostate-specific antigen.

${ }^{a} P$ values are from $\chi^{2}$ tests comparing the distribution of patients within each type of baseline characteristic between the sequenced/ unsequenced subgroups and the comparative overall population. $P>.05$ in all cases, demonstrating that the sampled populations do not differ significantly from the full patient population in terms of baseline characteristics. 
TABLE A3. Comparative Baseline Characteristics in Patients With Known Metastatic Burden

\begin{tabular}{|c|c|c|c|c|c|c|}
\hline Baseline Characteristic & $\begin{array}{l}\text { Sequenced } \\
(n=185)\end{array}$ & $\begin{array}{l}\text { Known Metastatic } \\
\text { Burden }(\mathrm{n}=94)\end{array}$ & $P^{a}$ & $\begin{array}{l}\text { Low Volume } \\
(n=40)\end{array}$ & $\begin{array}{l}\text { High Volume } \\
\quad(n=54)\end{array}$ & $P^{b}$ \\
\hline \multicolumn{7}{|l|}{ Age at randomization, years } \\
\hline Median & 69 & 70 & & 67 & 70 & \\
\hline IQR & $63-75$ & $63-74$ & & $62-74$ & $63-75$ & \\
\hline \multicolumn{7}{|l|}{ Presenting PSA, ng/mL } \\
\hline Median & 93 & 133 & & 62 & 292 & \\
\hline IQR & $25-393$ & $44-446$ & & $11-140$ & $81-1141$ & \\
\hline \multicolumn{7}{|l|}{ T stage } \\
\hline$\leq \mathrm{T} 2$ & $19(10)$ & $9(10)$ & \multirow[t]{4}{*}{.808} & $1(3)$ & $8(15)$ & \multirow[t]{4}{*}{.254} \\
\hline T3 & $102(55)$ & $47(50)$ & & $22(55)$ & $25(46)$ & \\
\hline $\mathrm{T} 4$ & $51(28)$ & 31 (33) & & $14(35)$ & $17(31)$ & \\
\hline Tx & $13(7)$ & $7(7)$ & & $3(8)$ & $4(7)$ & \\
\hline \multicolumn{7}{|l|}{ Nodal state } \\
\hline NO & $58(31)$ & $33(35)$ & \multirow[t]{3}{*}{.462} & $14(35)$ & $19(35)$ & \multirow[t]{3}{*}{.830} \\
\hline N1 & $115(62)$ & $52(55)$ & & $23(58)$ & $29(54)$ & \\
\hline $\mathrm{Nx}$ & $12(6)$ & $9(10)$ & & $3(8)$ & $6(11)$ & \\
\hline \multicolumn{7}{|l|}{ Disease category } \\
\hline De novo M1 & $182(98)$ & 93 (99) & \multirow[t]{2}{*}{.998} & 39 (98) & $54(100)$ & \multirow[t]{2}{*}{.880} \\
\hline Relapsed M1 & $3(2)$ & $1(1)$ & & $1(2)$ & $0(0)$ & \\
\hline \multicolumn{7}{|l|}{ Metastatic distribution } \\
\hline Bone only & $114(62)$ & $57(61)$ & \multirow[t]{3}{*}{.973} & $19(48)$ & $38(70)$ & \multirow[t]{3}{*}{$<.001$} \\
\hline Distant node only & $20(11)$ & $11(12)$ & & $11(28)$ & $0(0)$ & \\
\hline Bone and nodal or other & $51(28)$ & $26(28)$ & & $10(25)$ & $16(30)$ & \\
\hline \multicolumn{7}{|l|}{ Gleason } \\
\hline$\leq 7$ & $27(15)$ & $19(20)$ & \multirow[t]{3}{*}{.393} & $9(23)$ & $10(19)$ & \multirow[t]{3}{*}{.808} \\
\hline $8-10$ & $149(81)$ & $69(73)$ & & $28(70)$ & $41(76)$ & \\
\hline Unknown & $9(5)$ & $6(6)$ & & $3(8)$ & $3(6)$ & \\
\hline \multicolumn{7}{|l|}{ Performance status } \\
\hline 0 & $131(71)$ & $61(65)$ & \multirow[t]{3}{*}{.311} & $26(65)$ & $35(65)$ & \multirow[t]{3}{*}{.998} \\
\hline 1 & $52(28)$ & $33(35)$ & & $14(35)$ & $19(35)$ & \\
\hline 2 & $2(1)$ & $0(0)$ & & $0(0)$ & $0(0)$ & \\
\hline
\end{tabular}

NOTE. Data are presented as No. (\%) unless otherwise noted.

Abbreviations: IQR, interquartile range; PSA, prostate-specific antigen.

${ }^{a} P$ values are from $\chi^{2}$ tests comparing the distribution of patients within each type of baseline characteristic between the sequenced subgroup and the whole cohort with known metastatic burden.

${ }^{\mathrm{b}} P$ values are from $\chi^{2}$ tests comparing the distribution of patients within each type of baseline characteristic between low-and high-metastatic burden subgroups. As would be expected, patients differ only on the basis of metastatic distribution $(P<.001)$. 